Vladimir M. Shalaev

\title{
Nonlinear Optics of Random Media
}

Fractal Composites

and Metal-Dielectric Films

With 51 Figures 


\section{Contents}

1. Electromagnetic Properties of Random Composites.

A Review of Basic Approaches .................. 1

1.1 Introduction to Random Media: Fractals,

Self-Affine Surfaces and Percolation Composites .......... 1

1.2 Mean-Field Theories and Numerical Techniques .......... 5

1.3 Spectral Representation ....................... 9

1.4 Critical Behavior of Conductivity

and Dielectric Function in the Percolation Model ........ 10

2. Surface-Enhanced Nonlinear Optical Phenomena ....... 15

2.1 Introduction ............................. 15

2.2 Surface-Enhanced Optical Responses ............... 17

2.2.1 Kerr Optical Nonlinearity . . . . . . . . . . . . . . . 17

2.2 .2 Four-Wave Mixing $\ldots \ldots \ldots \ldots \ldots \ldots \ldots \ldots \ldots \ldots \ldots \ldots \ldots$

2.2.3 Raman Scattering ....................... 19

2.2.4 High-Order Harmonic Generation $\ldots \ldots \ldots \ldots \ldots \ldots, 19$

3. Small-Particle Fractal Aggregates ................ 21

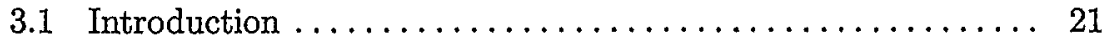

3.2 Basic Theoretical Approaches ..................... 24

3.2.1 Coupled Dipoles....................... 24

3.2.2 Polarizability of a Monomer ................. 26

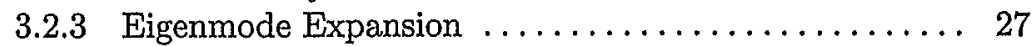

3.2.4 Quasi-Static Approximation .................. 29

3.3 Absorption Spectra of Fractal Aggregates.............. 33

3.4 Local-Field Enhancement in Fractals ............... 38

3.5 Near-Field Imaging and Spectroscopy of Hot Spots . . . . . . 41

3.6 Surface-Enhanced Optical Nonlinearities in Fractals......... 46

3.6.1 Qualitative Consideration and Estimates .......... 46

3.6.2 Enhanced Raman and Rayleigh Scattering......... 48

3.6.3 Nearly Degenerate Four-Wave Mixing ........... 53

3.6.4 Optical Kerr Effect ...................... 60

3.7 Local Photomodification in Fractals................ 64

3.8 Fractals in Microcavities ...................... 69 


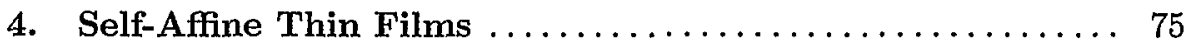

4.1 General Approach ............................... 75

4.2 Linear Optical Properties...................... 78

4.3 Enhanced Optical Phenomena on Self-Affine Surfaces ...... 81

4.3.1 Raman Scattering .................... 82

4.3.2 Second-Harmonic Generation ............... 84

4.3.3 Third-Harmonic Generation ................ 89

4.3.4 Kerr Optical Nonlinearity and Four-Wave Mixing..... 93

4.4 Nano-Optics of Fractal Aggregates Deposited on a Surface ... 95

4.4.1 Sample Preparation and Numerical Simulations ...... 95

4.4.2 Fractal-Surface-Enhanced Nonlinear Nano-Optics..... 96

5. Random Metal-Dielectric Films ................ 101

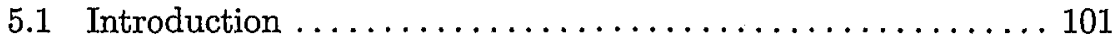

5.2 Giant Field Fluctuations $\ldots \ldots \ldots \ldots \ldots \ldots \ldots \ldots \ldots \ldots \ldots$

5.2.1 Linear Response......................... 105

5.2 .2 Nonlinear Response...................... 107

5.2 .3 Numerical Model ......................... 111

5.2 .4 Field Distributions ..................... 113

5.3 Scaling Theory for High-Order Field Moments .......... 118

5.3.1 Percolation and Anderson Transition Problem........ 118

5.3.2 Resonance Excitations at $\epsilon_{\mathrm{d}}=-\epsilon_{\mathrm{m}}^{\prime} \ldots \ldots \ldots \ldots \ldots 120$

5.3.3 Scaling Renormalization and Collective Resonances

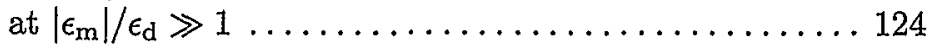

5.3.4 Comparison of Surface-Enhanced Phenomena in Fractal and Percolation Composites ........... 130

5.4 Some Experimental Results .................... 134

5.4.1 Near-Field Nano-Optics ..................... 134

5.4.2 Surface-Enhanced Raman Scattering........... 137

5.4.3 Kerr Optical Nonlinearity and White-Light Generation 139

5.5 Percolation-Enhanced Nonlinear Scattering ............ 141

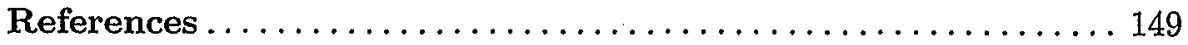

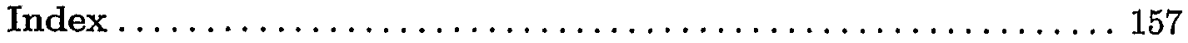

